\section{Lucienne Chatenoud Jean-François Bach}

ADRESSE

L. Chatenoud: docteur en médecine, docteur ès sciences, directeur de recherche à l'Inserm. J.F. Bach: docteur en médecine, docteur ès sciences, professeur d'immunologie à l'université René-Descartes, directeur de l'Inserm U. 25, chef du service d'immunologie clinique à l'hopital Nerker. Inserm U.25, hôpital Necker, 149, rue de Sèvres, 75743 Paris Cedex 15, France.

\title{
Peut-on rétablir la tolérance au soi dans les maladies auto-immunes?
}

Dans les pays industrialisés, les maladies auto-immunes (polyarthrite rhumatoïde, lupus érythémateux disséminé, sclérose en plaques, diabète juvénile) occupent le troisième rang après les maladies cardiovasculaires et le cancer. On comprend donc que leur diagnostic précoce et leur traitement demeurent des enjeux d'envergure. Au cours des dernières années, de nouvelles stratégies d'immunothérapie des maladies auto-immunes ont été proposées, dont le but est de restaurer de manière spécifique et durable la tolérance vis-à-vis du ou des autoantigène(s) pathogène (s). L'autoantigène pathogène, qui serait l'outil thérapeutique de choix, demeure très mal caractérisé dans la grande majorité des maladies auto-immunes humaines et ne peut donc être administré. L'utilisation transitoire de certains anticorps monoclonaux antilymphocytes, et plus particulièrement des anticorps anti-CD3, testée dans un modèle expérimental murin de diabète insulinodépendant, a permis d'induire une rémission totale et durable de l'atteinte auto-immune ; une voie thérapeutique nouvelle semble ainsi ouverte.

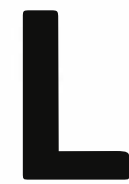

e système immunitaire de tout individu est physiologiquement tolérant au soi. En d'autres termes, il ne développe pas de réaction autoimmune susceptible d'induire des manifestations cliniques. Il existe, néanmoins, chez tout individu sain des lymphocytes autoréactifs reconnaissant les autoantigènes: les lymphocytes B porteurs de récepteurs immunoglobuliniques et/ou les lymphocytes $\mathrm{T}$ qui reconnaissent des antigènes peptidiques présentés par les molécules du complexe majeur d'histocompatibilité (CMH) [1]. Il se trouve, heureusement, que le développement de ces cellules B et $\mathrm{T}$ autoréactives, potentiellement pathogènes, est contrôlé par tout un réseau d'interactions cellulaires et moléculaires. Peut-être aussi les lymphocytes B et les lymphocytes T autoréactifs présents chez l'individu sain ou «naturels" ne sont-ils pas porteurs de récepteurs pouvant donner lieu à une réaction pathogène en raison d'une spécificité ou d'une affinité inadaptée. Seuls certains clones exprimant des récepteurs de forte affinité, tels qu'il en apparaît dans les processus auto-immuns pathologi- 


\section{RÉFÉRENCES}

1. Bach JF. La reconnaissance du soi et ses déréglements: les maladies auto-immunes. Auto-immunité physiologique et pathologique. In: Bach JF, ed. Traité d'immunologie. Paris: Flammarion, 1993 : 751-86.

2. Kappler JW, Roehm N, Marrack P. T cell tolerance by clonal elimination in the thymus. Cell 1987; 49: 273-80.

3. Schwartz RH. Immunological tolerance. In: Paul WE, ed. Fundamenlal immunology. New York: Raven Press, 1993: 677-731.

4. Billingham RE, Brent L, Medawar PB. Actively acquired tolerance to foreign cells. Nature $1953 ; 172$ : 603-6.

5. Posselt AM, Barker CF, Tomaszewski JE, Markmann JF, Choti MA, Naji A. Induction of donor-specific unresponsiveness by intrathymic islet transplantation. Srience 1990; $249: 1293-5$

6. Odorico JS, Barker CF, Posselt AM, Naji A. Induction of donor-specific tolerance to rat cardiac allografts by intrathymic inoculation of bone marrow. Surgery 1992; 112 : $370-6$.

7. Sykes M, Sheard M, Sachs DH. Effects of $T$ cell depletion in radiation bone marrow chimeras. I. Evidence for a donor cell population which increases allogeneic chimerism but which lacks the potential to produce GVHD. J Immunol 1988; 141 : 2282-8.

8. Zinkernagel RM, Althage A, Callahan G, Welsh Rm JR. On the immunocompetence of H-2 incompatible irradiation bone marrow chimeras. I Immunol $1980 ; 124$ : 235665.

9. Sykes M, Sachs DH. Bone marrow transplantation as a means of inducing tolerance. Semin Immunol 1990; 2: 401-17.

10. Monaco AP, Wood ML, Russell PS. Studies on heterologous antilymphocyte serum in mice. III. Immunological tolerance and chimerism produced across the H2-locus with adult thymectomy and antilymphocyte serum. Ann NY Acad Sci 1966; 129: 190-209.

11. Wood ML, Monaco AP, Gozzo IJ, Liegeois A. Use of homozygous allogeneic bone marrow for induction of tolerance with antilymphocyte serum: dose and ti- ques, pourraient être capables d'induire des lésions d'organe source de manif estations cliniques. Il s'agit là d'une question fondamentale, malheureusement non encore résolue, puisque, à quelques exceptions près, on ne sait pas vraiment distinguer un lymphocyte autoréactif pathogène d'un lymphocyte autoréactif qui ne l'est pas.

Par ailleurs, il existe probablement une tolérance beaucoup plus absolue vis-à-vis de certains autoantigènes, secondaire à la sélection dite négative des lymphocytes $\mathrm{T}$ spécifiques dans le thymus. On sait, en effet, que l'expansion des cellules autoréactives reconnaissant dans le thymus les molécules du $\mathrm{CMH}$, éventuellement associées à des peptides du soi, est souvent suivie de leur élimination par apoptose [2]. Cette sélection négative par apoptose concerne plus particulièrement les lymphocytes qui reconnaissent les antigènes du soi exprimés dans le thymus (ainsi que certains rétrovirus, qui entraînent une délétion sélective des lymphocytes T porteurs de certains fragments $\mathrm{V} B \mathrm{du}$ récepteur des cellules $\mathrm{T}$ ). La nature des autoantigènes qui donnent lieu à une telle sélection négative demeure mal définie. Sans doute incluent-ils certaines molécules fortement représentées dans l'organisme, notamment les molécules ubiquitaires, telles que les protéines du cytosquelette ou certaines protéines sériques présentes à forte concentration, comme l'albumine.

Tel qu'il fut initialement défini dans les années 1960, le concept de tolérance concerne exclusivement les états de «non réponse " ou "paralysie " immunitaire, observés à la place de l'immunisation habituelle, lors de l'administration d'un antigène selon des protocoles particuliers. Un des exemples les plus classiques est la tolérance aux gammaglobulines humaines observée, chez la souris adulte, après injection intraveineuse de gammaglobulines débarrassées de tout agrégat. Non seulement les souris ne produisent pas d'anticorps dirigés contre les gammaglobulines humaines désagrégées, mais elles deviennent réfractaires à toute immunisation par cet antigène, même lorsque les immunoglobulines sont inoculées en présence d'adjuvant complet de Freund, c'est-à-dire dans des conditions où, normalement, elles donnent lieu à une très forte immunisation [3]. De façon semblable, on avait, à la suite des travaux de Medawar [4], défini la tolérance d'allogreffe autour de l'observation très remarquable, et inattendue, que l'injection à des souris nouveau-nées de cellules spléniques allogéniques (provenant d'une souche au CMH incompatible), non seulement n'entraîne pas de réponse immunitaire vis-à-vis des antigènes portés par les cellules spléniques, mais induit, au contraire, une paralysie immunitaire durable et spécifique des alloantigènes "tolérogènes". Ainsi, ces souris devenues adultes ne rejettent pas les greffes de peau provenant de la même souche que celle ayant fourni les cellules spléniques injectées à la naissance, alors qu'elles rejettent normalement les allogreffes d'une tierce partie [4].

On avait d'abord pensé, à l'instar de ce qui était observé et bien démontré pour les lymphocytes B, que ces phénomènes de tolérance, qui impliquent très largement les cellules $\mathrm{T}$, étaient dus à la disparition, ou délétion, des cellules $T$ spécifiques des antigènes tolérogènes. Cette conception de la tolérance a progressivement évolué. On s'est d'abord aperçu que, dans bien des cas, les cellules reconnaissant l'antigène tolérogène ne disparaissaient pas mais étaient devenues "anergiques", c'est-à-dire incapables d'une réponse immunitaire efficace vis-à-vis de l'antigène, alors qu'elles demeurent sensibles à une stimulation polyclonale [3]. Et puis, surtout, plus récemment, on a réalisé que, dans un grand nombre de cas, y compris dans les modèles de tolérance néonatale, la paralysie immunitaire est en fait un phénomène actif, lié à l'apparition de cellules $\mathrm{T}$ régulatrices qui bloquent la différenciation des cellules $\mathrm{T}$ effectrices ou leurs effets biologiques [3].

Désormais, il faut donc envisager une définition plus large de la tolérance immunitaire, sans préjuger des mécanismes en cause, qui incluent les phénomènes d'immunorégulation. De façon pratique, on peut définir la tolérance comme l'absence des manifestations biologiques de la réaction immunitaire induite par un antigène, en dehors d'une immunosuppression 
généralisée. Celle-ci peut, néanmoins, être nécessaire pour induire la tolérance, qu'il s'agisse d'immaturité immunitaire du nouveau-né ou d'administration transitoire, chez le sujet adulte, de produits immunosuppresseurs. Il est important d'insister sur le fait que, chez l'adulte, l'état de tolérance persiste longtemps après la fin des traitements immunosuppresseurs qui ont permis son induction.

Dans le cas qui nous intéresse ici, il s'agira donc de rétablir la tolérance au soi, défaillante dans les maladies auto-immunes, sans chercher nécessairement à supprimer toute réponse immunitaire vis-à-vis du soi, qu'elle implique les lymphocytes B ou les lymphocytes $\mathrm{T}$.

\section{Induction de tolérance vis-à-vis des antigènes extrinsèques}

De très nombreuses études ont été consacrées depuis cinquante ans à la recherche de méthodes permettant d'induire la tolérance. Une des méthodes les plus efficaces reste le recours au modèle du nouveau-né [4]. On peut induire une tolérance vis-àvis de divers antigènes, chez l'animal nouveau-né dont le système immunitaire n'est que très partiellement formé. Il faut pour cela intervenir dans les premiers jours de la vie chez la souris. La question s'est posée un moment de savoir si la technique pourrait être extrapolable à l'homme dans les premiers jours de la vie. Rien n'est moins sûr car il s'avère que dans des espèces autres que la souris, notamment chez le mouton, le moment limite d'administration de l'antigène pour induire une tolérance peut précéder la naissance. Il est bien sûr difficile de se prononcer pour ce qui concerne l'espèce humaine. On voit mal, du point de vue thérapeutique, comment avoir recours à cette stratégie chez des enfants nouveaunés, avec peut-être l'exception de certaines greffes d'organes chez des enfants présentant de très graves malformations viscérales, notamment cardiaques ou hépatiques.

De façon générale, l'administration à forte dose, par voie intraveineuse, de molécules solubles est peu immunogénique et induit volontiers la tolérance, comme nous l'avons vu dans l'exemple des gammaglobulines hu- maines désagrégées chez la souris [3]. Une des explications serait que, au moins pour les lymphocytes B, lorsqu'un antigène leur est présenté à forte concentration, au lieu de se différencier en cellules produisant des anticorps, ce lymphocyte devient anergique.

On peut aussi introduire l'antigène tolérogène dans un site privilégié comme le thymus pour faciliter la sélection négative $[5,6]$. Cette méthode a été appliquée avec succès pour des allogreffes d'îlots de Langerhans [5].

On peut enfin, et cela est apparemment la méthode la plus à notre portée en thérapeutique humaine, avoir recours à l'administration combinée de l'antigène et d'immunosuppresseurs puissants, donnés pendant une courte période. C'est ainsi que l'on peut induire une tolérance chez les animaux auxquels on injecte des cellules allogéniques provenant, par exemple, de la moelle osseuse, en association avec un traitement par sérum antilymphocyte ou une irradiation lymphoïde totale [7-12]. De façon intéressante et un peu inattendue, il semble que l'adjonction simultanée d'un traitement par anticorps antilymphocytes monoclonaux et d'une greffe de peau ou mieux d'organe, notamment de cœur, facilite l'induction de tolérance spécifique d'alloantigènes [13-21]. Les mécanismes de cette tolérance induite sous couvert d'immunosuppresseurs, en particulier les anticorps monoclonaux (anti-CD3, anti-CD4), ne semblent faire intervenir ni délétion, ni anergie, mais plutôt une tolérance active, telle qu'elle a été décrite précédemment.

Une autre méthode consiste à administrer l'antigène per os [22]. C'est une observation bien connue depuis toujours que l'administration d'antigènes dans l'alimentation induit très rarement une immunisation. Il existe, certes, des allergies digestives mais celles-ci restent relativement peu fréquentes. Au contraire, il semblerait que l'introduction d'antigènes dans l'alimentation, pour peu que les antigènes ou des peptides qui en dérivent soient suffisamment protégés des altérations liées à leur passage dans le tube digestif, puisse induire une tolérance. La stimulation dans l'intestin de certaines cellules à fonction suppressive qui, dans un second temps, quitteraient le tube digestif pour gagner les organes lymphoïdes pourrait expliquer cette tolérance.

Tolérance dans les modèles de maladies auto-immunes provoquées par l'administration d'autoantigènes

Il est possible d'induire toute une série de maladies auto-immunes en injectant des préparations de divers organes ou, mieux, des antigènes purifiés à partir de ces organes, en présence d'un adjuvant, par exemple l'adjuvant complet de Freund [1]. On a ainsi décrit une encéphalomyélite allergique expérimentale induite par injection de protéine basique de la myéline, une thyroïdite expérimentale induite par l'injection de thyroglobuline et une myasthénie expérimentale induite par l'injection de préparations purifiées de récepteur de l'acétylcholine $\left(\mathrm{m} / \mathrm{s} n^{\circ} 11\right.$, vol. 1), p. 1177). Des efforts importants ont été consacrés à la recherche de méthodes pouvant conduire à la prévention spécifique de l'apparition de ces maladies, en induisant un phénomène de tolérance.

Ainsi, l'injection de thyroglobuline soluble à des souris non auto-immunes prévient l'induction ultérieure de la thyroïdite expérimentale après injection de thyroglobuline dans des conditions induisant normalement la thyroïdite (thyroglobuline en présence d'adjuvant). De même, on peut empêcher l'apparition d'une encéphalomyélite allergique expérimentale en injectant la protéine basique de la myéline par voie intraveineuse en présence d'adjuvant incomplet de Freund, avant l'administration de la protéine en présence d'un adjuvant conduisant à la maladie (adjuvant complet de Freund, Bordetella pertussis). Il est également possible de prévenir l'induction de l'encéphalite en injectant des fragments de la protéine, dont il a été préalablement montré qu'ils étaient insuffisants pour induire la maladie mais pouvaient, néanmoins, en assurer sa prévention. Enfin, il est possible de prévenir l'encéphalomyélite allergique expérimentale par l'administration de protéine basique de la myéline per os, selon le modèle qui a été décrit plus haut [22]. 
Tolérance induite chez la souris NOD, le modèle animal du diabète humain insulino-dépendant

\section{- Le diabète de la souris NOD}

La souris non obese diabetic (NOD) est une souris de souche pure qui développe après 4 à 6 mois, plus fréquemment chez la femelle que chez le mâle, un diabète insulino-dépendant en tout point semblable au diabète humain de type I [23-26]. La maladie commence vers la quatrième semaine par l'apparition d'une infiltration de cellules mononucléées autour, puis dans les îlots de Langerhans (l'insulite). L'insulite devient progressivement invasive puis destructrice. Lorsqu'une fraction suffisante de cellules $\beta$ a été détruite, la maladie clinique apparaît. ('ette destruction est complétée par une inflammation réversible qui accélère l'évolution de la maladie. I e diabète est de type autoimmun, il est transféré à des souris non diabétiques par des cellules $\mathrm{T}$ purifiées (il faut à la fois des cellules CD4 et des cellules (:D8) [27, 28]. Il peut être prévenu par un très grand nombre de manipulations inactivant ou détruisant sélectivement les cellules T. I a maladie est génétiquement contrôlée et de nombreux gènes de prédisposition ont été identifiés [24, 26, 29-33], en premier lieu les antigènes du $\mathrm{CMH}$, mais aussi de nombreux gènes situés en dehors du $\mathrm{CMH}$.

Si le rôle des cellules $T$ est bien démontré - il a été confirmé par la mise en évidence des effets diabétogènes de clones de cellules $\mathrm{T}(\mathrm{m} / \mathrm{s}$ $n^{\circ} 8$-9, vol. 9, p. 998) et par l'apparition du diabète chez des souris transgéniques exprimant le récepteur $\mathrm{T}$ de tels clones - les mécanismes du diabète restent dans une large mesure encore incertains [34]. L'antigène cible n'est pas connu avec certitude même s'il existe d'excellents candidats, en particulier l'insuline et la glutamic acid decarboxylase (GAD) $(\mathrm{m} / \mathrm{s}$ $n^{\circ}$ 9, vol. 6, p. 921) [35-37]. La nature des mécanismes effecteurs reste également mal définie. Le rôle des cellules CD4 produisant les cytokines, qui attirent sur place les macrophages, reste l'hypothèse la plus plausible sans pour autant éliminer le rôle potentiel cytotoxique direct de cellules de type CD8 [24-26].

\section{- Prévention de la maladie}

En appliquant les concepts précédents à la souris NOD, il est apparu logique d'essayer de prévenir le diabète, soit en injectant par voie intraveineuse l'un des antigènes candidats, et cela a été réalisé avec succès avec la GAD, soit en administrant par voie orale ces antigènes, notamment la GAD et surtout l'insuline $(\mathrm{m} / \mathrm{s}$ $n^{\circ} 12$, vol. 9, p. 1432) [38, 39]. On peut aussi empêcher l'apparition du diabète par la greffe de cellules d'îlots syngéniques dans le thymus de souris NOD jeunes [26]. Toutes ces méthodes sont efficaces et leur intérêt théorique est évident. Il faut néanmoins remarquer qu'elles n'entraînent la prévention de la maladie que lorsqu'elles sont appliquées très tôt dans la vie, dans le premier mois. Le problème est alors de savoir quel est le degré de spécificité de ces interventions puisque de très nombreuses manipulations du système immunitaire, beaucoup moins spécifiques que celles qui viennent d'être évoquées, sont également capables de prévenir la maladie lorqu'elles sont utilisées chez la souris jeune. C'est en particulier le cas de l'interleukine 1 , du poly-IC, du nicotinamide et de certains régimes hypocaloriques ou dépourvus en protéine du lait [26].

L’interprétation de ces expériences est, en outre, compliquée par deux phénomènes de mieux en mieux compris, mais qui viennent brouiller les cartes, l'extension de la sensibilisation et la suppression de voisinage. Dans le premier cas, il n'est fait référence à l'observation que lorsque la souris NOD se sensibilise contre un antigène des cellules $\beta$, la sensibilisation s'étend à d'autres motifs antigéniques qui leur sont propres soit par des phénomènes d'interaction moléculaire (epitope spreading) [38], soit par des phénomènes d'extension intermoléculaire. Ainsi, l'inflammation induite par la première réaction immunitaire, notamment la production d'interféron $\gamma$, augmente l'expression de certaines molécules dont celles du CMH qui augmente l'immunogénicité de la cible. Alternativement, la dégradation des cellules, induite par la première attaque, provoque une sensibilisation secondaire contre les produits de dégradation. Le résultat final est
21. Wood KJ. Transplantation tolerance with monoclonal antibodies. Semin Immunot 1990 ; 2 : 389-99. 
qu'il est extrêmement difficile de définir le caractère primitif ou secondaire des différents phénomènes observés. Quant au phénomène de suppression de proximité, bien décrit dans le modèle de la tolérance orale, il fait allusion au fait que, dans certains cas, les cellules suppressives dirigées contre un antigène des cellules $\beta$ sécrètent des cytokines qui vont agir à proximité et supprimer les réactions immunitaires dirigées contre d'autres antigènes des cellules $\beta$ que ceux contre lesquels les cellules productrices de ces cytokines sont elles-mêmes dirigées.

Tolérance induite chez la souris NOD vis-à-vis des antigènes d'îlots par administration d'anticorps monoclonaux anti-CD3

Il était connu depuis plusieurs années que l'administration de divers anticorps monoclonaux dirigés contre les antigènes de membrane des cellules $\mathrm{T}$ (récepteur des cellules $\mathrm{T}, \mathrm{CD} 3$, CD4, récepteur de l'IL2...) prévenait l'apparition du diabète lorsque le traitement était réalisé avant le deuxième mois [26]. Nous avions nous-mêmes montré qu'un traitement par un anticorps anti-CD3 pouvait également empêcher l'apparition du diabète chez les souris mâles qui font peu ou pas de diabète spontanément mais qui le développent très régulièrement après injection de cyclophosphamide [40]. Le mécanisme d'action de ces anticorps monoclonaux était sans doute, dans ces cas-là, voisin de celui des autres immunosuppresseurs comme la ciclosporine, via l'inhibition de la fonction des cellules $T$ ou leur déplétion.

L'idée est venue, à partir des travaux réalisés dans les modèles de transplantation, d'utiliser les anticorps monoclonaux chez des souris NOD ayant atteint un stade beaucoup plus avancé de leur maladie, au moment où le diabète vient d'apparaître, comme en témoignent l'élévation de la glycémie et l'apparition d'une glycosurie [40]. Même si un pourcentage important des cellules $\beta$ est alors détruit, il en reste encore un nombre suffisant pour maintenir un équilibre métabolique convenable après régression de l'inflammation qui, nous l'avons dit, se surajoute à la destruc- tion des cellules $\beta$. L'existence d'une telle inflammation explique la normalisation de l'hyperglycémie, obtenue en quelques heures chez les souris récemment devenues diabétiques par l'administration d'un anticorps monoclonal antirécepteur des cellules $T$, qui n'agit que sur les cellules $T$ sans toucher directement les autres cellules mononucléées infiltrant le pancréas [41]. On peut également citer la récupération importante de la production d'insuline par les îlots de souris NOD devenues récemment diabétiques après culture des îlots in vitro en l'absence des lymphocytes ou du sérum de ces souris [42].

Les anticorps anti-CD3 représentaient un choix intéressant puisqu'il est bien établi cliniquement que ces anticorps sont parmi les plus puissants immunosuppresseurs que l'on connaisse aujourd'hui. Les anticorps anti-CD3, plus particulièrement l'OKT3, sont d'ailleurs les seuls anticorps monoclonaux utilisés en routine clinique en transplantation et cela depuis maintenant plus de dix ans [43]. Rappelons que la molécule CD3 représente, en fait, un complexe de chaînes polypeptidiques (l'anticorps est dirigé contre la chaîne $\varepsilon$ ), étroitement lié, bien que de façon non covalente, au récepteur T. Le traitement par l'anticorps CD3 fait disparaître les cellules $\mathrm{CD}^{+}$, non pas tellement par destruction (déplétion), mais plutôt par modulation antigénique, c'est-à-dire redistribution du récepteur CD3 (et du récepteur $\mathrm{T}$ qui lui est associé) de la surface de la cellule puis internalisation ou externalisation (shedding) (figure 1) [44, 45]. Au-delà de ce mécanisme conduisant à l'immunosuppression, les anticorps anti-CD3 induisent une très forte activation des lymphocytes $\mathrm{T}$ dont témoigne, in vivo, la libération massive de différentes cytokines (TNF $\alpha$ d'origine lymphocytaire, IL2, interféron $\gamma$, IL4, IL6, IL10, GM-CSF...) [46-48].

Le schéma expérimental initial a consisté à suivre l'histoire naturelle des souris NOD en recherchant régulièrement l'apparition d'une glycosurie puis d'une hyperglycémie. Chez les souris diabétiques, cinq injections d'un anticorps anti-CD3 produit chez le hamster ont été administrées de façon quotidienne pendant cinq jours à la dose de $5 \mu \mathrm{g}$ (suffisante pour exercer un effet immunosuppresseur mais insuffisante pour déclencher des effets secondaires graves par libération de cytokines) [40]. Dans un premier temps, on assiste à la régression du diabète, ce qui n'est pas très surprenant étant donné les résultats qui ont été évoqués plus haut avec d'autres anticorps et, de façon plus générale, avec les immunosuppresseurs, encore que, à ce stade avancé, nombre d'immunosuppresseurs aient perdu leur activité. Fait plus surprenant et faisant tout l'intérêt de ce modèle, il s'est avéré que les rémissions induites par l'anticorps antiCD3 étaient durables, quasi définitives, sans tendance à la récidive [40].

Le problème s'est immédiatement posé du mécanisme de l'induction d'une telle rémission. Le rôle de l'activité mitogène de l'anticorps, qui provoque la libération de cytokines après la première injection, put être rapidement éliminé puisque l'administration de fragments $F(a b ') 2$ de l'anticorps, qui n'activent pas les lymphocytes $\mathrm{T}$ car dépourvus de fragment Fc, provoque le même état de tolérance (Tableau I). On peut également éliminer un effet de déplétion strict parce que l'anticorps anti-CD3 n'entraîne pas de déplétion durable des lymphocytes au-delà de deux semaines après la fin du traitement.

On se retrouve donc, pour expliquer ces résultats, devant les trois possibilités évoquées plus haut, à savoir la délétion, l'anergie ou l'induction d'une tolérance active. Les deux premières hypothèses furent rapidement éliminées. La persistance d'une insulite de type périphérique chez les animaux tolérants rendait improbable un mécanisme de délétion: on voyait mal, en effet, si cette délétion avait représenté le mécanisme essentiel de la tolérance, comment les lymphocytes $\mathrm{T}$ auraient persisté dans les îlots [40]. La preuve directe qu'il existait toujours chez ces souris des cellules $T$ potentiellement diabétogènes fut apportée par deux expériences. D’une part, le traitement des souris tolérantes par le cyclophosphamide, qui est connu pour sa capacité de détruire les cellules $\mathrm{T}$ suppressives à fort index de prolifération, se révéla capable de "casser" la tolérance, c'est-à-dire de faire apparaître le diabète chez les souris tolérantes. D'autre part, lorsque les cellules spléniques des souris tolérantes furent injectées à des sou- 


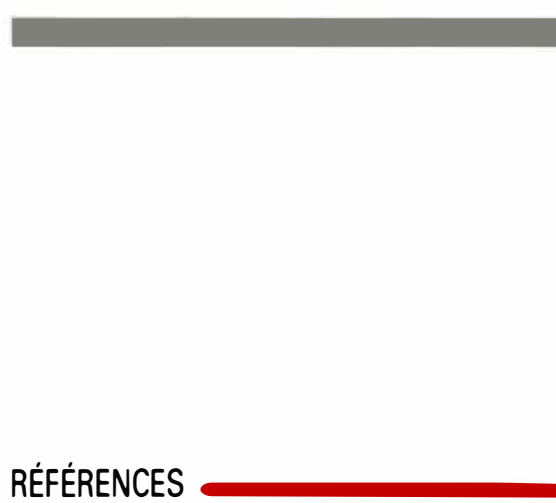

22. Weiner HL, Zhang ZI, Khoury SJ, Miller A, Al-Sabbagh A, Brod SA, Lider O, Higgins P, Sobel R, Nussenblatt RB, Haffler D. Antigen-driven peripheral immune tolerance. Suppression of organ-specific autoimmune diseases by oral administration of autoantigens. Ann NY Acad Sci 1991 ; 636: 227-32.

23. Makino S, Kunimoto K, Muraoka Y, Mizushima Y, Katagiri K. Tochino Y. Breeding of a non-obese, diabetic strain of mice. $k x p$ Anim 1980; 29 : 1-13.

24. Boitard C, Bach J. Pathogénie du diabète insulino-dépendant, maladie polygénique d'origine auto-immune. méderine/sriences 1991 ; $7: 226-34$.

25. Honeyman MC, Harrison LC. The immunologic insult in type 1 diabetes. Springer Semin Immunopathol 1993; 14 : 253-74.

26. Bach JF. Insulin-dependent diabetes mellitus as an autoimmune disease. Endorrnol Rev 1994; 15: 516-42.

27. Matsumoto M, Yagi H, Kunimoto K, Kawaguchi J, Makino S, Harada M. Transfer of autoimmune diabetes from diabetic NOD mice to NOD athymic nude mice: the roles of $\mathrm{T}$ cell subsets in the pathogenesis. Cell Immunol 1993; 148: 189-97.

28. Christianson SW, Shultz LD, Leiter EH. Adoptive transfer of diabetes into immunodeficient NOD-scid/scid mice. Relative contributions of $\mathrm{CD} 4^{+}$and $\mathrm{CD} 8^{+}$T-cells from diabetic versus prediabetic NOD.NON-Thy1 a donors. Diabetes $1993 ; 42: 44-55$.

29. Todd JA, Aitman TJ, Cornall RJ, Ghosh $S$, Hall JR, Hearne CM, Knight AM, Love JM, McAlleer MA, Prins JB, Rodrigues N, Lathrop M, Pressey A, Delarato NH. Peterson LB, Wicker LS. Genetic analysis of autoimmune type 1 diabetes mellitus in mice. $\mathrm{Na}$ ture $1991 ; 351: 542-7$.

30. Garchon HJ, Bedossa P, Eloy L, Bach JF Identification and mapping to chromosome 1 of a susceptibility locus for periinsulitis in non-obese diabetic mice. Nature 1991; 353 . 260-2.

31. Wicker LS, Appel MC, Dotta F, Pressey A, Miller BJ, Delarato NH, Fischer PA, Boltz Rc IR, Peterson L.B. Autoimmune syndromes in major histocompatibility com plex (MHC) congenic strains of nonobese diabetic (NOD) mice. The NOD MHC: is dominant for insulitis and cyclophosphamide-induced diabetes. J Exp Med 1992; 176 :

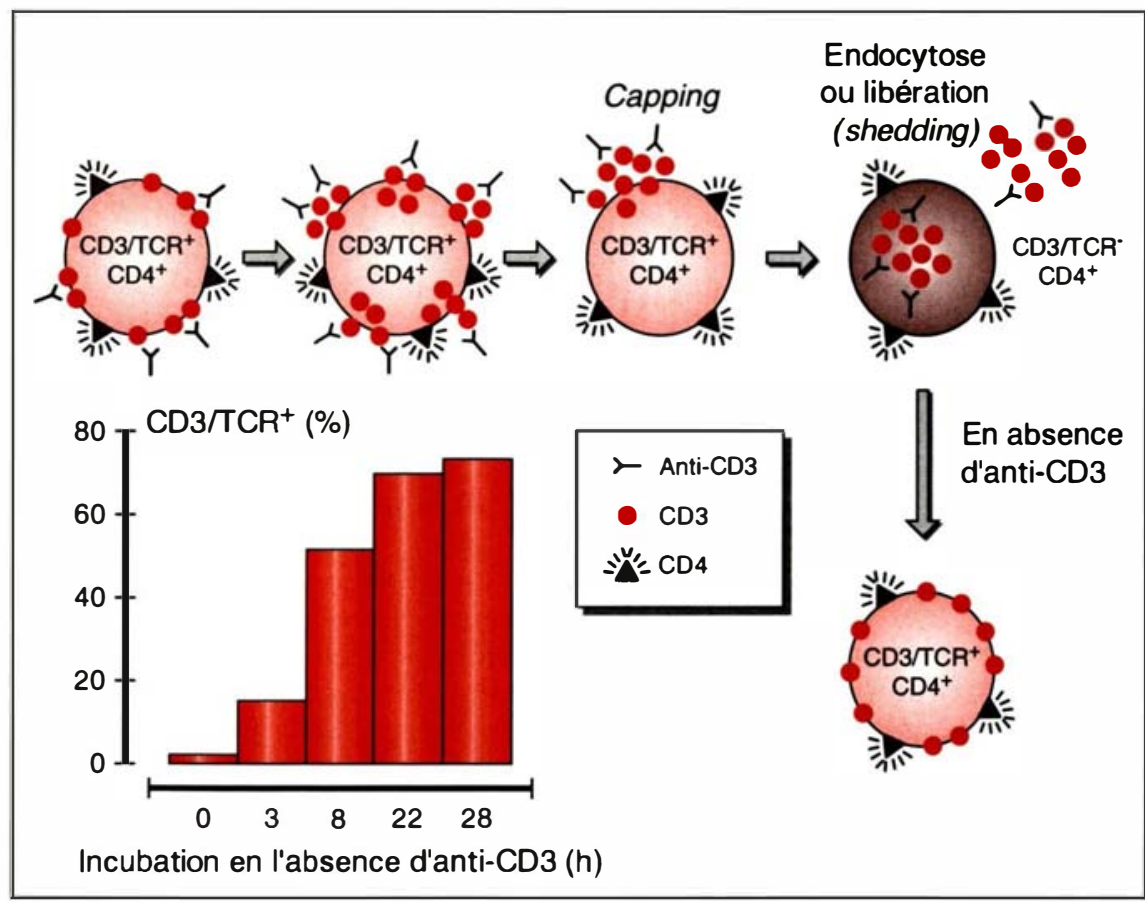

Figure 1. Mécanismes d'action cellulaire des anticorps anti-CD3 sur les lymphocytes $T$. Représentation schématique du phénomène de modulation antigénique illustré ici pour un lymphocyte $\mathrm{CD}^{+}$. Dans les minutes qui suivent la fixation d'un ligand spécifique (ici l'anticorps monoclonal anti-CD3) à son récepteur de surface cellulaire (ici la molécule CD3), on observe la redistribution des complexes ainsi formés et leur concentration à un pôle de la cellule (phénomène dit de capping). Les complexes sont ensuite internalisés dans la cellule ou libérés dans le milieu environnant (phénomène dit de shedding). Ainsi, la cellule lymphocytaire en question perd de manière transitoire l'expression de la molécule $C D 3$ et du récepteur $T$ de reconnaissance de l'antigène qui lui est intimement associé. Il est fondamental de souligner que le phénomène de modulation antigénique est spécifique du récepteur qui est la cible de l'anticorps utilisé. De ce fait, comme le montre le schéma, l'expression des récepteurs autres que CD3/TCR (illustrée ici par le cas de CD4) n'est pas significativement modifiée. La modulation antigénique est un phénomène réversible: lorsque l'anticorps disparaît du milieu environnant, on assiste à une réexpression rapide et totale d'un complexe CD3/TCR fonctionnel à la surface du lymphocyte $T$ Il'histogramme illustre la cinétique de cette réexpression à la surface de lymphocytes $T$ circulants, prélevés chez un patient traité par un anticorps monoclonal anti-CD3 (OKT3), et incubés in vitro en l'absence de l'anticorps monoclonal). (D'après [45]).

ris syngémiques irradiées, un diabète apparut chez les receveurs ( $\mathrm{L}$. Chatenoud et al., résultats non publiés). Il restait donc comme explication principale la tolérance active. Encore fallait-il en préciser les modalités. Utilisant un modèle de cotransfert précédemment décrit dans le laboratoire [49], dans lequel on montre que les cellules de souris NOD prédiabétiques ont la capacité de suppri- mer l'effet diabétogène des cellules spléniques provenant de souris diabétiques, nous avons essayé de mettre en évidence les cellules suppressives chez les souris rendues tolérantes par l'anticorps anti-CD3. Une telle démonstration n'a pu être apportée pour l'instant. Cela s'explique sans doute par le fait que les souris rendues tolérantes par l'anti-CD3 n'ont pas une quantité de cellules protec- 


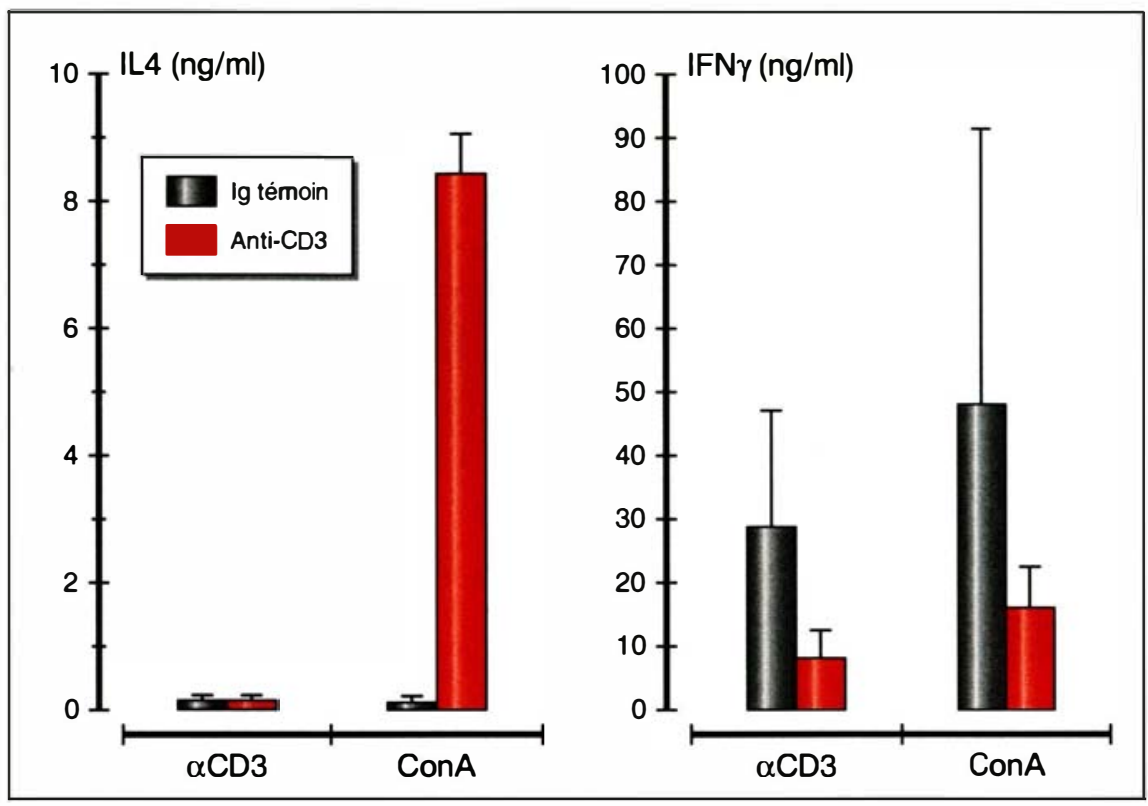

Figure 2. Production de cytokines par les cellules spléniques des souris NOD traitées par anti-CD3. Chez des souris NOD femelles traitées par l'anticorps monoclonal de hamster 145 2C11, spécifique de la chaîne $\varepsilon d u$ complexe CD3 murin, ou des immunoglobulines normales de hamster, les cellules spléniques ont été prélevées deux semaines après traitement. Les splénocytes ainsi obtenus ont été cultivés in vitro en présence de deux mitogènes, l'anticorps anti-CD3 ou la concanavaline $A$. Au terme de 48 heures de culture, les surnageants ont été prélevés et leur contenu en interleukine 4 (cytokine de type TH2) et en interféron $\gamma$ (IFN $\gamma$ ) (cytokine de type TH1) a été dosé grâce à un test immunoenzymatique (ELISA). On note, chez les animaux ayant reçu I'anti-CD3, une augmentation len présence de concanavaline $A$, ConAl de production d'interleukine 4 et une diminution de celle d'interféron $\gamma$.

Tableau I

INCIDENCE DE LA RÉMISSION DU DIABĖTE INSULINODÉPENDANT OBSERVÉE CHEZ LES SOURIS NOD TRAITEES PAR ANTI-CD3

\begin{tabular}{|cccc|}
\hline & Anti-CD3 & $\begin{array}{c}\text { Fragments F(ab') } 2 \\
\text { de l'anti-CD3 }\end{array}$ & $\begin{array}{c}\text { Ig polyclonales de } \\
\text { hamster (Ig témoins) }\end{array}$ \\
\hline $\begin{array}{c}\text { \% rémission } \\
\text { (12 semaines après la fin } \\
\text { du traitement) }\end{array}$ & $\begin{array}{c}78 \\
(n=45)\end{array}$ & 64 & 0 \\
$(n=58)$ & $(n=17)$ \\
\hline
\end{tabular}

Les souris NOD femelles ont été traitées au moment de l'apparition du diabète (glycosurie positive et glycémie > $4 \mathrm{~g} / \mathrm{ll}$ ) selon l'un des protocoles suivants: le premier groupe a reçu l'anticorps anti-CD3 (145 2C11, Ig de hamster, 5 à $20 \mu g$ i.v./jour pendant 5 jours consécutifs); le deuxième groupe les fragments $\mathrm{F}\left(\mathrm{ab} \mathrm{b}^{\prime}\right) 2$ de l'anticorps (50 $\mathrm{\mu g}$ i.v./jour, 5 jours consécutifs) et le troisième groupe des lg témoins (lg polyclonales de hamster 5 à $50 \mu g$ i.v./jour. 5 jours consécutifs). La rémission a été définie comme le retour à une normoglycémie stable et durable et l'absence de glycosurie (i.v.: voie intraveineuse).

trices suffisante pour qu'on puisse les révéler dans le système de transfert qui semble donner un avantage important aux cellules diabétogènes, également présentes, nous venons de le voir, dans la rate des souris tolérantes. Peut-être aussi les cellules protectrices sont-elles localisées dans le pancréas et est-ce là qu'il faudrait les rechercher plutôt que dans la rate. Une autre façon d'aborder le problè- me est de rechercher un déséquilibre dans la production des cytokines immunorégulatrices. On sait, en effet, qu'il existe deux catégories de cytokines : celles produites par les cellules THl (essentiellement l'interleukine 2 et l'inferféron $\gamma$ ) qui sont responsables de l'immunité à médiation cellulaire et les cytokines produites par les cellules TH2 (ensentiellement IL4, IL.5 et IL.10) qui sont respon- sables de l'effet auxiliaire vis-à-vis de la production des anticorps [50]. Fait essentiel, au-delà de leurs fonctions propres, les cytokines de chacune de ces deux populations ont un effet de régulation négative sur l'autre population cellulaire. Ainsi, les cytokines de type TH2 (notamment l'IL 4 et l'IL 10) peuvent-elles supprimer les fonctions THl qui conduisent aux réactions d'hypersensibilité retardée, 


\section{RÉFÉRENCES}

32. Bohme I, Schuhbaur B, Kanagawa $O$, Benoist C, Mathis D. MHC-linked protection from diabetes dissociated from clonal deletion of T cells. Science 1990; 249: 293-5.

33. Hattori M, Buse JB, Jackson RA, Glimcher L, Dorf ME, Minami M, Makino S, Moriwaki $\mathrm{K}$, Kuzuya $\mathrm{H}$, Imura $\mathrm{H}$, Strauss $\mathrm{WM}$, Seidman JG, Eisenbarth GS. The NOD mouse: recessive diabetogenic gene in the major histocompatibility complex. Science $1986 ; 231: 733-5$.

34. Haskins K, McDuffie M. Acceleration of diabetes in young NOD mice with a $\mathrm{CD} 4^{+}$islet-specific T cell clone. Science 1990; 249: $1433-6$.

35. Baekkeskov S, Aanstoot HJ, Christgau S, Reetz A, Solimena M, Cascalho M, Folli F, Richter-Olesen $\mathrm{H}$, de Camilli P. Identification of the $64 \mathrm{~K}$ autoantigen in insulin-dependent diabetes as the GABA-synthesizing enzyme glutamic acid decarboxylase. Nature $1990 ; 347 ; 151-6$.

36. Palmer JP, Asplin CM, Clemons P, Lyen $K$, Tatpati $O$, Raghu PK, Paquette TL. Insulin antibodies in insulin-dependent diabetics before insulin treatment. Science 1983 ; 222: $1337-9$

37. Froguel $P$. Un nouveau gène du diabète insulino-dépendant sur le chromosome $\mathrm{llq}$ Le DID, paradigme pour l'étude des maladies multifactorielles. médecine/sriences 1994; $10: 1147-9$

38. Kaufman DL, Clare-Salzler M, Tian J, Forsthuber T, Ting GSP, Robinson P, Atkinson MA, Sercarz EE, Tobin AJ, Lehmann PV. Spontaneous loss of T-cell tolerance to glutamic acid decarboxylase in murine insulin-dependent diabetes. Nature 1993; 366: 69-72.

39. Tisch R, Yang XD, Singer SM, Liblau RS, Fugger L, McDevitt HO. Immune response to glutamic acid decarboxylase correlates with insulitis in non-obese diabetic mice. Nature $1993 ; 366$ : 72-5.

40. Chatenoud L, Thervet E, Primo J, Bach JF. Anti-CD3 antibody induces long-term remission of overt autoimmunity in nonobese diabetic mice. Proc Nall Acad Sci USA 1994; 91: 123-7.

41. Sempe P, Bedossa P, Richard MF, Villa MC, Bach JF, Boitard C. Anti-alpha/beta T cell receptor monoclonal antibody provides an efficient therapy for autoimmune diabetes in nonobese diabetic (NOD) mice.
42. Strandell E, Eizirik DL, Sandler S. Reversal of beta-cell suppression in vitro in pancreatic islets isolated from nonobese diabetic mice during the phase preceding insulin-dependent diabetes mellitus. J Clin Invest 1990; 85: 194450 .

43. Cosimi AB, Colvin RB, Burton RC, Rubin RH, Goldstein G, Kung PC, Hansen WP, Delmonico FL, Russell PS. Use of monoclonal antibodies to T-cell subsets for immunologic monitoring and treatment in recipients of renal allografts. NEngl JMed 1981 ; 305: 308-14.

44. Chatenoud L, Baudrihaye MF, Kreis $\mathrm{H}$ Goldstein G, Schindler J, Bach JF. Human in vivo antigenic modulation induced by the anti-T cell OKT3 monoclonal antibody. Eur J Immunol 1982; 12 : 979-82.

45. Chatenoud L, Bach JF. Antigenic modulation: a major mechanism of antibody action. Immunol Today 1984; 5: 20-5.

46. Ferran C, Sheehan K, Dy M, Schreiber R, Merite S, Landais P, Noel LH, Grau G, Bluestone J, Bach JF, Chatenoud L. Cytokine-related syndrome following injection of anti-CD3 monoclonal antibody: further evidence for transient in vivo $\mathrm{T}$ cell activation. EurJ Immunol 1990; 20: 509-15.

47. Chatenoud L, Ferran C, Legendre C, Thouard I, Merite S, Reuter A, Gevaert Y Kreis $\mathrm{H}$, Franchimont $\mathrm{P}, \mathrm{Bach}$ JF. In vivo cell activation following OKT3 administration. Systemic cytokine release and modulation by corticosteroids. Transplantation 1990; 49: 697-702.

48. Abramowicz D, Schandene L, Goldman $M$, Crusiaux A, Vereerstraeten $\mathrm{P}$, de Pauw L, Wybran J, Kinnaert P, Dupont E, Toussaint C. Release of tumor necrosis factor, interleukin 2, and $\gamma$-interferon in serum after injection of OKT3 monoclonal antibody in kidney transplant recipients. Transplantation $1989 ; 47: 606-8$.

49. Boitard C, Yasunami R, Dardenne M, Bach JF. T cell-mediated inhibition of the transfer of autoimmune diabetes in NOD mice. J Exp Med 1989; 169: 1669-80.

50. Mosmann TR, Coffman RL. THl and TH2 cells : different patterns of lymphokine secretion lead to different functional properties. Annu Rev Immunol 1989; 7: 145-73.

51. Bolt S, Routledge E, Lloyd I, Chatenoud L, Pope H, Gorman SD, Clark M, Waldmann $\mathrm{H}$. The generation of a humanized, non-mitogenic CD3 monoclonal antibody which retains in vitro immunosuppressive properties. Eur J Immunol 1993; 23: 403-11. au rejet des greffes et, très probablement, à l'apparition du diabète chez la souris NOD. En l'absence de la connaissance de l'autoantigène cible du diabète, qui nous permettrait d'étudier directement la production des cytokines TH1 et TH2 par les lymphocytes $T$ diabétogènes, nous avons étudié, chez les souris traitées par anti-CD3, la production des cytokines de type THl et TH2 par l'ensemble des cellules $T$. De fait, après une injection d'anticorps anti-CD3, nous avons observé en réponse à un mitogène tel que la concanavaline $A$, une tendance à l'augmentation de la production d'IL4 et une réduction de la production d'interféron $\gamma$, en comparaison avec des souris non traitées par l'anticorps (figure 2). Il reste à prouver que ce nouvel équilibre induit par l'anticorps est durable.

\section{Conclusion et conséquences thérapeutiques}

Cette nouvelle approche de l'immunothérapie des maladies auto-immunes, fondée sur la restauration de la tolérance aux autoantigènes, ouvre des perspectives considérables, surtout si, comme nous venons de le montrer, elle s'applique à des malades présentant des signes cliniques et non pas seulement au stade de la prédisposition génétique, avant que les mécanismes auto-immuns se soient mis en route. Les méthodes utilisant les autoantigènes sont sans doute les plus simples et n'impliquent pas l'induction d'une immunosuppression, aussi courte soit-elle. Mais, nous l'avons dit, il est très difficile dans le diabète, comme dans la plupart des maladies auto-immunes humaines, de cerner avec précision l'autoantigène responsable de la maladie, si tant est qu'il en existe un seul. La stratégie que nous proposons est de réalisation beaucoup plus simple et ne nécessite pas le recours à l'autoantigène. Elle pose cependant le problème de la disponibilité d'un anticorps monoclonal non activateur. Il semble, en effet, préférable d'utiliser un anticorps ne provoquant pas la libération massive de cytokines. L'utilisation de tels anticorps est acceptable en transplantation, étant donné le contexte clinique et l'utilisation conjointe d'immuno-suppresseurs 
qui diminuent l'activation, mais inacceptable dans la pratique des maladies auto-immunes où la préactivation des cellules $\mathrm{T}$ et l'absence de traitement associé rendent cet effet secondaire très sévère. La question se pose de savoir s'il faudra utiliser des fragments $F\left(a b^{\prime}\right) 2$, comme nous l'avons fait chez la souris NOD, ou plutôt des anticorps monoclonaux humanisés $\left(m / s n^{\circ} 6 / 7\right.$, vol. 1), p. 748) utilisant des fragments Fc ne donnant pas lieu à l'activation. De tels anticorps ont été produits [51]. Ils pourraient être utilisés chez l'homme.

La question pourrait être posée du risque éventuel de l'effet immunosuppresseur associé à l'administration de ces anticorps monoclonaux, surtout si l'on s'adresse à des patients au début de leur maladie. Dans le type de protocole que nous avons utilisé chez la souris NOD, ce risque paraît mineur dans la mesure où le traitement est de courte durée, c'est-à-dire très insuffisant pour exposer au risque de "surimmunosuppression" surtout en l'absence de traitement associé. On sait, en effet, d'après l'expérience acquise en transplantation, que seuls les traitements par l'OKT3 de plus de quinze jours, associés à de fortes doses d'autres immunosuppresseurs, exposent au risque de surimmunosuppression (inf ection à germe opportuniste, lymphomes).

Couplée au diagnostic précoce de la maladie, une telle stratégie pourrait permettre, à terme, une prévention efficace, durable et sans danger du diabète insulinodépendant

\section{INSTITUT \\ POUR LA RECHERCHE SUR LA MOELLE ÉPINIËRE}

\section{SUBVENTOHS DE RECYERGYE D'UN MONTANT ANNUEL \\ DE 150000 F A 200000 F CHACUNE reconductibles au maximum deux fois}

Destinées à financer des projets présentés par des Laboratoires de Recherche français

\section{Thème proposé}

Lésions de la moelle épinière notamment étude des lésions traumatiques expérimentales et évaluation clinique chez l'homme des manifestations neurovégétatives

\section{Comité scientifique}

M. le Docteur BESSON, M. le Professeur HURTH M. le Professeur AGID, M. le Professeur BEDOISEAU, M. le Professeur DEBURGE,

M. le Docteur DOLLFUS, M. le Professeur GRANT, M. le Professeur LAPORTE.

M. le Docteur MAURY, M. le Professeur RAISMAN

\section{Constitution du dossier} de candidature

Les candidats doivent demander par écrit les formulaires nécessaires au Secrétariat du Conseil Scientifique de l'Association

\section{Obligations :}

Les candidats s'engagent à : - rédiger un rapport d'activité annuel - citer l'Institut pour la Recherche sur la Moelle Épinière en signature de toutes publications et communications inhérentes aux travaux effectués

Clôture du dépôt des dossiers : 31 janvier 1996

\section{IRME}

45, rue Vineuse - 75116 Paris Tél. : (1) 44.05 .15 .43

\begin{tabular}{|c|c|c|c|}
\hline & $\begin{array}{r}\text { SOCIÉTÉ I } \\
\text { Collège c } \\
\text { Journée C } \\
22 \text { nOVE }\end{array}$ & lbr & $\begin{array}{l}\text { IOLOGIE } \\
\text { Paris } \\
\text { Bernard } \\
\text { Pe } 1995\end{array}$ \\
\hline & $\begin{array}{l}\text { Thérap } \\
\text { (Tronsgénèse, vecteurs viroux e }\end{array}$ & & $\begin{array}{l}\text { nique } \\
\text { Premiers essais de thérapie) }\end{array}$ \\
\hline $\begin{array}{l}8 \mathrm{~b} 45 \\
9 \mathrm{~b} 10 \\
9 \mathrm{bSO} \\
10 \mathrm{~b} 30 \\
11 \mathrm{~b} 15 \\
11 \mathrm{~b} 46 \\
14 \mathrm{~b} 00\end{array}$ & 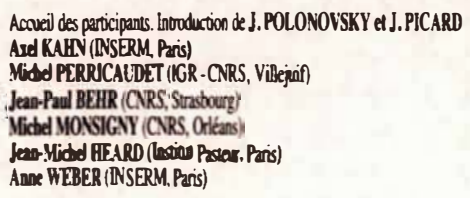 & $\begin{array}{l}14 \mathrm{~b} 30 \\
15 \mathrm{~b} 00 \\
15 \mathrm{~b} 30 \\
16 \mathrm{~b} 30 \\
17 \mathrm{~b} 00 \\
17 \mathrm{~h} 6\end{array}$ & 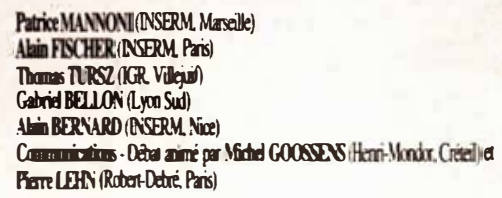 \\
\hline & $\begin{array}{r}\text { Inscription pour le. } \\
\text { auprès du Secrétariar de la Société de Biologie. Collège }\end{array}$ & & $\begin{array}{l}\text { ations et le lunch: } \\
\text { 3. rue d'Ulm. } 75231 \text { Paris Cedex 05 ( } 8 \text { 44.27.13.40) }\end{array}$ \\
\hline
\end{tabular}

\section{Summary}

Restoring self tolerance in autoimmune diseases ?

Pathologic autoimmune responses may be responsible for a wide variety of diseases including rheumatoid arthritis, systemic lupus erythematosus, multiple sclerosis and insulin dependent diabetes mellitus. Since in developed countries autoimmune diseases represent the third greatest cause of morbidity after cardio-vascular diseases and cancer, defining tools for successful therapy remains a major challenge. Treatments are generally non specific and require the chronic administration of immunosuppressive or anti-inflammatory drugs whose side effects are far from negligible. In some cases such as insulin dependent diabetes, substitutive replacement therapies alone are used (namely insulin) due to our inability to treat the autoimmune process. Over the last few years, new immunointervention strategies have been proposed for autoimmune diseases, with the aim of restoring self-tolerance in a durable manner. Until recently, the dogma was that this specifc immunotherapy could only result from the administration of the exogenous autoantigen, the practical application of which is difficult in human autoimmune diseases since the target autoantigen is usually unknown. Another interesting possibility is the usage of anti-T lymphocyte antibodies. Using a murine model of diabetes, we recently showed that a short treatment with a monoclonal antibody to $\mathrm{CD} 3$ could induce total and permanent remission of spontaneous established autoimmune diabetes. The immune mechanisms responsible for the anti-CD3 effect as well as the potential clinical application of this therapeutic approach are discussed.

TIRÉS À PART 